\title{
Serological diagnosis of $\mathbf{Q}$ fever endocarditis
}

\author{
G. Dupuis*, O. Péter*, R. Lüthy†, J. Nicoletł, M. Peacock§ and W. Burgdorfer§
}

* Division of Clinical Microbiology and Infectious Diseases, Valais Central Institute, Sion, $\dagger$ Division of Infectious Diseases, University Hospital, Zürich, $\ddagger$ Institute of Veterinary Bacteriology, University of Bern, Bern, Switzerland and \$LLaboratory of Pathobiology, Rocky Mountain Laboratories, National Institute of Allergy and Infectious Diseases, Hamilton, Montana, U.S.A.

KEY WORDS: Q fever, Coxiella burnetii, rickettsial endocarditis.

The diagnosis of $Q$ fever endocarditis cannot be made by bacterial cultures and necessitates serological identification of specific antibodies to Coxiella burnetii which stimulates mainly the production of anti-phase II antibodies during the acute diséase, but primarily anti-phase I antibodies in endocarditis. Indirect microimmunoftuorescence allows rapid detection of specific $\mathrm{Ig} A, \operatorname{lgG}$ and $\mathrm{IgM}$. The results of serological analyses of 191 acute cases of $Q$ fever were compared with those of 8 cases of Coxiella burnetii endocarditis. All sera were evaluated by complement fixation and microimmunofluorescence tests. The highest titre differences between primary $Q$ fever and $Q$ fever endocarditis were observed with anti-phase I IgA and IgG antibodies measured by microimmunofluorescence followed by anti-phase I antibodies measured by complement fixation tests. Antiphase IIgG and IgM titres were consistently higher than anti-phase II titres in endocarditis. The reverse is true in acute $Q$ fever. In addition, anti-phase IIgA appeared to be diagnostic for Coxiella burnetii endocarditis. Accordingly we recommend the testing of these specific $\operatorname{Ig} A$, $\operatorname{IgG}$, and $\lg M$ by microimmunofluorescence in cases of culture-negative endocarditis. These tests could also prove useful for following the development of Coxiella burnetii endocarditis in patients under treatment.

\section{Introduction}

Primary or acute $Q$ fever is an influenza-like infection, frequently accompanied by pneumonia. It normally follows a benign course, although progression to a chronic stage, Coxiella burnetii endocarditis, may occur and presents a dangerous complication ${ }^{[l]}$. Although only about 200 cases have been reported so far, $Q$ fever endocarditis appears to occur more frequently than assumed. Most cases have been discovered in the United Kingdom and Australia ${ }^{[2-4]}$.

The frequency of endocarditis after primary $Q$ fever is estimated to be in the order of 1 to $2 \%$, but so far no work has been done to confirm this. The latency period between the acute or primary phase, which has of ten gone undiagnosed, and endocarditis is still unknown, with estimates ranging from 2 to 20 years. Although more often diagnosed in middleaged men, $Q$ fever endocarditis can also affect women and children ${ }^{(5)}$. Clinically, it presents itself

Submitted for publication on the 6 May 1986.

Address for correspondence: G. Dupuis, Valais Central Institute, $\mathrm{CH}-195$ I Sion 3/Switzerland. as a culture-negative endocarditis, with patients suffering from fever, tachycardia, heart murmur, hepatomegaly and splenomegaly. Often there is a history of pre-existing mitral or aortic valvular lesion ${ }^{[6,7]}$. Nonspecific laboratory examinations often reveal a leucocytosis with anaemia and thrombopenia, hypergammaglobulinaemia and pathologic liver tests ${ }^{[8]}$. With a mortality rate of up to $50 \%$, even with medical and surgical treatment, it is imperative to diagnose these cases at the earliest possible time to shorten delay of effective antibiotic therapy. Isolation of $C$. burnetii from routine blood cultures is not possible, and only serology allows a clinical diagnosis. Inoculation of guinea pigs with valvular tissues taken during surgery frequently allows a definite confirmation of the diagnosis.

Unique to $C$. burnetii is its antigenic phase variation. The virulent phase $I$ is isolated from natural or laboratory infections of animals and man, whereas the avirulent phase II occurs during serial passage of $C$. burnetii in immunologically incompetent hosts, such as embryonated hen eggs or cell tissue culture systems. Phase I to II variation seems to correlate with smooth and rough lipopolysaccharide changes ${ }^{[9]}$. Specific high levels of anti-phase I antibodies are normally found in chronic $Q$ fever 
(endocarditis), whereas specific anti-phase II antibodies predominate during primary $Q$ fever.

The serological test most commonly used up to the present time has been the complement fixation (CF) test, with anti-phase I antibody titres greater than 1:200 being considered diagnostic of $C$. burnetii endocarditis. However, cases have been discovered with titres below this leve[i']. Among the more sensitive serological tests developed during the last few years, is the microimmunofluorescence test (IFA), which allows the rapid and easy detection of specific IgA, IgG and IgM antibodies to phase I and phase II C. burnetii ${ }^{[10]}$.

This paper deals with a comparative serological analysis of the CF and IFA tests in 8 cases of Q fever endocarditis and in 191 cases of acute $Q$ fever ${ }^{[11]}$.

\section{Materials and methods}

The sera of the 8 patients suffering from culturenegative endocarditis were obtained from the Departments of Internal Medicine of the University Hospitals at Zürich and Berne and from the Pediatric Service of the University Hospital at Geneva. The diagnosis of $Q$ fever endocarditis for each of these cases was established between 1980 and 1984 according to the classically known criterion of an anti-phase I antibody titre greater than 1:200 in CF. The IFA analyses (IgA, IgG, IgM, to $C$. burnetii phases I and II) were performed from frozen serum specimens in late 1984 . Only results of the first sample from each patient, i.e. before any antibiotic therapy, are reported here.

A short description of the patients condition at the time of the diagnosis is presented. Patient 1 was admitted for surgical correction of a ventricular septal defect. Follow-up clinical investigation revealed an endocarditis with fever, hepatosplenomegaly, and negative blood cultures. A liver biopsy showed a granulomatous hepatitis. Guinea pigs inoculated with the patient's blood developed antibodies to $C$. burnetii. The patient's serum showed high CF anti-phase I antibody titre. Patients 2 and 3 were admitted for a fever of unknown origin, and with aortic regurgitation. Investigations revealed an endocarditis, negative blood cultures and a high

- CF anti-phase I antibody titre. Patient 4 was admitted for surgical correction of a tetralogy of Fallot. Clinical investigation revealed an endocarditis with

- fever, hepatosplenomegaly, negative blood cultures and a high CF anti-phase I antibody titre. Patient 5 underwent an aortic valve replacement for stenosis in November 1979. In June 1982, he was admitted for fever of unknown origin. The follow-up clinical investigations revealed endocarditis, negative blood cultures and a high CF anti-phase I antibody titre. He was treated with cotrimoxazole and rifampicin. In January 1983, he underwent a second aortic valve replacement for regurgitation. $C$. burnetii was isolated from heart valve tissue. Patient 6 was admitted for a fever of unknown origin. Follow-up clinical investigations revealed an endocarditis, negative blood cultures and a high CF anti-phase I antibody titre. Patient 7 presented a serologically confirmed acute $Q$ fever in 1973 . He underwent a first aortic valve replacement for regurgitation in 1977 and a second in April 1981. He was admitted in November 1981 for a fever of unknown origin with heart failure. Follow-up clinical investigations revealed an endocarditis, negative blood cultures and a high CF anti-phase I antibody titre. Patient 8 underwent in January 1985 an aortic, mitral and tricuspid valve replacement for rheumatic heart disease. Clinical investigations revealed an endocarditis with fever, negative blood cultures and a high CF anti-phase I antibody titre.

The 191 sera from patients with acute $Q$ fever were sent to us by physicians in Le Châble during an epidemic in the Val de Bagnes (Valais) in the autumn of 1983. All 191 sera were examined by CF and IFA (IgA, IgG, IgM) tests for anti-phase I and II antibodies to $C$. burnetiil ${ }^{[12.13]}$. Sera of 12 patients suffering from streptococcal endocarditis were also examined for these antibodies.

\section{Complement fixation test (CF)}

The micro-method was performed as described by the Centers for Disease Control (CDC, Atlanta, GA, U.S.A) ${ }^{[14]}$ except that the starting serum dilution was $1: 10$ and the sensitized erythrocyte concentration was $2.5 \%$. The antigen $C$. burnetii phase I was of strain Herzberg (Veterinaria AG, Zürich, Switzerland) and the antigen $C$. burnetii phase II was of strain Nine Mile (Virion AG, Rüschlikon, Switzerland). Serum, antigen, complement (Virion AG) and haemolytic system (Boehring, Marburg, Germany), were prepared in veronal buffer (Oxoid BR 16). The end point was defined as the highest dilution with $\geqslant 75 \%$ fixation. Results are expressed as reciprocal titres (for example 200 for the $1: 200$ dilution).

\section{Indirect microimmunofluorescence test (IFA)}

The IFA tests were performed according to the 
Table 1 Reciprocal C. burnetii titres in patients with Q-fever endocarditis obtained with the complement fixation test and the microimmunofluorescence test using antigens of phase I and II

\begin{tabular}{|c|c|c|c|c|c|c|c|c|c|}
\hline \multirow{3}{*}{ Patient } & \multicolumn{2}{|c|}{ Complement fixation } & \multicolumn{6}{|c|}{ Microimmunofluorescence } & \multirow{3}{*}{$\begin{array}{l}\text { Rheumatoid } \\
\text { factor }\end{array}$} \\
\hline & \multirow{2}{*}{ I } & \multirow{2}{*}{ II } & \multicolumn{2}{|c|}{ IgG } & \multicolumn{2}{|c|}{ IgM } & \multicolumn{2}{|c|}{$\operatorname{IgA}$} & \\
\hline & & & I & II & I & II & I & II & \\
\hline 1 & 640 & 160 & 40960 & 640 & 320 & 20 & 160 & 20 & + \\
\hline 2 & 320 & 1280 & 20480 & 20480 & 640 & 640 & 1280 & 40 & + \\
\hline 3 & 1280 & 1280 & 40960 & 20480 & 640 & 320 & 2560 & 320 & + \\
\hline 4 & 640 & 640 & 20480 & 2560 & 80 & 80 & 160 & 20 & + \\
\hline 5 & 640 & 20 & 20480 & 2560 & 640 & 40 & 160 & 1280 & + \\
\hline 6 & 640 & 1280 & 40960 & 20480 & 640 & 320 & 320 & 20 & + \\
\hline 7 & 640 & 640 & 20480 & 5120 & 160 & 80 & 2560 & 160 & + \\
\hline 8 & 640 & 640 & 20480 & 5120 & 160 & 20 & 320 & 1280 & + \\
\hline Average & 679 & 742 & 28160 & 9680 & 410 & 188 & 940 & 390 & + \\
\hline
\end{tabular}

method described by Philip et al. ${ }^{[15]}$. The C. burnetii phase I antigen (Nine Mile strain) had been passed 307 times in guinea pigs, cloned from primary chicken embryo cell culture plaques and passed twice in chicken embryos. The C. burnetii phase II antigen (strain Nine Mile) had been passed 90 times in chicken embryos, cloned from primary chicken embryo cell culture plaques and passed three times in chicken embryos. Antigens of $C$. burnetii phase I and II were applied side by side to microscope slides with a pen over a premarked 18-dot template. We used FITC (fluorescein isothiocyanate) goat antihuman IgM and IgG conjugates (Biomérieux, France), and rabbit anti-human IgA conjugate (Dakopatts, Denmark). IgM reactive sera were tested for rheumatoid factor with the rheumatoid factor tube test 'Roche' (Roche Diagnostica, Basel, Switzerland).

\section{Results}

Table I shows individual and average titres for sera taken at the moment of diagnosis from the 8 cases of $C$. burnetii endocarditis. Phase I CF titres were consistently above 200 , with an average titre of 679. Anti-phase I IgG titres by IFA were consistently higher with an average titre of 28160 .

Figure 1 illustrates the quantitative relationship between the average titres for the 191 cases of acute $Q$ fever and for the 8 cases of $C$. burnetii endocarditis. The highest titre differences between primary $Q$ fever and $Q$ fever endocarditis were observed with anti-phase I IgA and IgG antibodies measured by
IFA, followed by anti-phase I antibodies measured by CF.

Table 2 compares the range of titres obtained with the two tests for the 8 cases of endocarditis and the 191 cases of acute $Q$ fever. Antibodies to phase $I$ in CF as well as anti-phase I IgA and IgG in IFA tests were never present at significant levels in primary $Q$ fever but were very high in endocarditis. An overlap of elevated titres was regularly observed in phase II titres. Phase I IFA titres were always equal or superior to phase II titres except for IgA in cases 5 and 8 . Rheumatoid factor was positive in all 8 cases.

In the 12 sera from patients suffering from streptococcal endocarditis, no anti-phase I or II IgA or IgM against $C$. burnetii were detected. In the sera from 3 patients only residual anti-phase I and II IgG were found. No rheumatoid factor was detected.

\section{Discussion}

Antibody titres to C. burnetii phase I, measured by $\mathrm{CF}$, were always greater than 200 in all eight cases of endocarditis. In contrast, none of the 191 cases of primary $Q$ fever presented a titre greater than 20 . This confirms the validity of the diagnostic criterion of phase I antibody titres in the diagnosis of $Q$ fever endocarditis. However, titres of less than 200 , do not exclude this diagnosis ${ }^{[1]}$. Antibody titres to $C$. burnetii phase II by CF cannot distinguish endocarditis from primary $Q$ fever because they are often at the same level. 


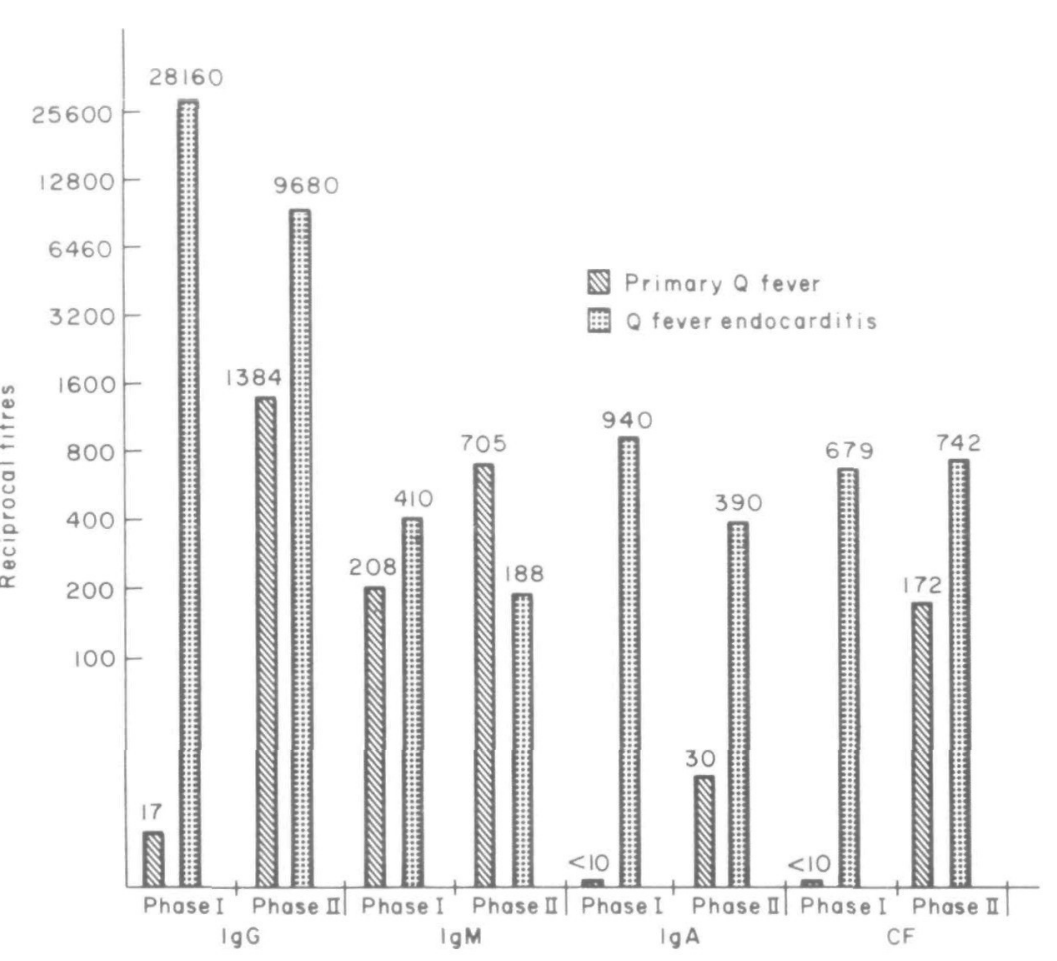

Figure I Average reciprocal C. burnetii titres in patients with primary $\mathrm{Q}$ fever (191 cases) and $Q$ fever endocarditis ( 8 cases) obtained with the complement fixation test (CF) and microimmunofluorescence test (IgG, IgM, IgA) using antigens of phase I and II.

Table 2 Range of reciprocal $\mathrm{C}$. burnetii titres in patients with $Q$ fever endocarditis and primary $Q$-fever obtained with the complement fixation test $(C F)$ and the microimmunofuorescence test (IFA) using antigens of phase I and II

\section{Serologic test}

Endocarditis $(N=8)$

Primary $\mathrm{Q}$ fever $(N=191)$

\section{CF I}

CF II

IFA IgG I

IgG II

IFA IgM I

IgM II

IFA IgA I

IgA II

Rheumatoid factor
$<10-20$

40-1280

$<20-40$

$320-10240$

$<20-640$

$80-10240$

$<20$

$<20-320$

$<20$

With IFA, anti-phase I IgG antibodies can be detected at very high dilutions in endocarditis only; the average titre for the 8 cases was approximately 28000 whereas it was 17 in the 191 cases of acute $Q$ fever. Anti-phase I IgG appears to be the most

sensitive discriminator, since none of the 8 cases of endocarditis had a level below 20480 , whereas none of the 191 cases of primary $Q$ fever had a level above 40. Because anti-phase II IgG are raised in both the acute and the chronic disease state, they are of no differential diagnostic value. Moreover, in each individual case, anti-phase I IgG titre was always equal or higher than anti-phase II IgG titre in endocarditis, and greatly lower in primary $Q$ fever. Our results correlate well with those reported by Peacock et al. ${ }^{[10]}$ for their 5 cases of $Q$ fever endocarditis.

With IFA, anti-phase I IgM are usually present at slightly higher levels than those of anti-phase II IgM in cases of endocarditis; the reverse is true in acute cases. However, due to the presence of rheumatoid factors in cases of $C$. burnetii endocarditis, the clinical significance of these IgM titres is uncertain. On the other hand, the rheumatoid factor being absent in acute $Q$ fever, IgM is diagnostic in that case $^{[12]}$.

Elevated anti-phase I IgA titres were found in all 8 patients and appear to be diagnostic for $C$. burnetii endocarditis. In contrast, such titres were 
undetectable in the 191 cases of acute $Q$ fever. On the other hand, anti-phase II IgA antibodies in variable titres were found in both clinical conditions as reported by others ${ }^{[16]}$. Consequently, only antiphase I IgA is a useful indicator for suspected cases of C. burnetii endocarditis.

No significant levels of IgA, IgG and IgM anti-C. burnetii phase I or II were found in the sera of the 12 persons with streptococcal endocarditis.

In addition to the CF test, the clinician can now use the IFA test, a sensitive, rapid and simple method, to establish the diagnosis of $C$. burnetii endocarditis. In accordance with Peacock et al. ${ }^{[10]}$ we found that the most sensitive parameters for the diagnosis of $C$. burnetii endocarditis are anti-phase I IgA and IgG. Another possible advantage of these indicators identified by IFA would be their earlier appearance in sera from patients developing a $C$. burnetii endocarditis. Regular checks of all the cases of acute $Q$ fever we diagnosed in 1983 will possibly confirm this. The level of these indicators during control checks of cases of $C$. burnetii endocarditis under treatment could also prove useful.

We would like to thank the cooperation of M. P. Glauser (CHUV, Lausanne), D. Laufer (Hôpital Cantonal, Genève), F. Burkhardt (Universität Bern, Bern), M. F. Paccaud (Institut d'Hygiène, Genève), L. Matter (Mikrobiologie, St-Gall) and M. C. Mottiez (ICHV, Sion). This work was supported by a grant from the Swiss Foundation for Cardiology.

\section{References}

[1] Turck WPG, Howitt G, Turnberg LA et al. Chronic Q fever. QJ Med 1976; 45(178): 193-217.

[2] Palmer SR, Young SEJ. Q fever endocarditis in England and Wales, 1975-81. Lancet 1982; 2(8313): 1448-9.
[3] Ellis ME, Smith CC, Moffat MAJ. Chronic or fatal Q-fever infection: a review of 16 patients seen in NorthEast Scotland (1967-80). QJ Med 1983; 52(205): 54-66.

[4] Wilson HG, Neilson GH, Galea EG, Stafford G, O'Brien MF. Q fever endocarditis in Queensland. Circulation 1976; 53(4): 680-4.

[5] Jones RWA, Pitcher DW. Q fever endocarditis in a 6 year old child. Arch Dis Child 1980; 55(4): 312-5.

[6] Haldane EV, Marrie TJ, Faulkner RS el al. Endocarditis due to $Q$ fever in Nova Scotia: experience with five patients in 1981-1982. J Infect Dis 1983; 148(6): 978-85.

[7] Varma MPS, Adgey AAJ, Connolly JH. Chronic Q fever endocarditis. Br Heart J 1980; 43(6): 695-9.

[8] Tobin MJ, Cahill N, Gearty Get al. $Q$ fever endocarditis. Am J Med 1982; 72(3): 396-400.

[9] Baca OG, Paretsky D. Q Fever and Coxiella burnetii: a Model for host-parasite interactions. Microbiol Rev 1983; 47: 127-49.

[10] Peacock MG, Philip RN, Williams JC, Faulkner RS. Serological evaluation of $Q$ fever in humans: enhanced phase I titres of immunoglobulins $G$ and $A$ are diagnostic for $Q$ fever endocarditis. Infect Immun 1983; 41(3): 1089-98.

[11] Dupuis G, Péter O, Pedroni D, Petite J. Aspects cliniques observés lors d'une épidémie de 415 cas de fièvre $Q$ Schweiz med Wochenschr 1985; 115: 814-8.

[12] Péter O, Dupuis G, Burgdorfer W, Peacock $M$. Evaluation of the complement fixation and indirect immunofluorescence tests in the early diagnosis of primary Q fever. Eur J Clin Microbiol 1985; 4: 394-6.

[13] Dupuis G, Péter O, Peacock M, Burgdorfer W, Haller E. Immunoglobulin responses in acute $Q$ fever. J Clin Microbiol 1985; 22(4): 484-7.

[14] Palmer DF. Complement fixation test. In Rose NR, Friedman $\mathrm{H}$, eds. Manual of clinical immunology, 2nd ed. Washington, DC: American Society for Microbiology, 1980: 35-47.

[15] Philip RN, Casper EA, Ormsbee RA, Peacock MG, Burgdorfer $W$. Microimmunofluorescence test for the serological study of Rocky Mountain spotted fever and typhus. J Clin Microbiol 1976; 3: 51-61.

[16] Worswick D, Marmion BP. Antibody responses in acute and chronic $Q$ fever and in subjects vaccinated against $Q$ fever. J Med Microbiol 1985; 19: 281-96. 